\title{
A nonlinear control method for the electromagnetic suspension system of the maglev train
}

\author{
Junqi XU*, Yuan ZHOU \\ Shanghai Maglev Transportation Engineering R\&D Center, Shanghai 201204, China
}

\begin{abstract}
To deal with the inherent nonlinearity and open-loop instability of the electromagnetic suspension (EMS) system, a new nonlinear control method is proposed. The simulation results show that, for a PID controller, the overshoot of the system response to an airgap step disturbance is about $3 \mathrm{~mm}$, and the transient time is $6 \mathrm{~s}$; however, for the proposed nonlinear controller, there is no overshoot and transient time within $2 \mathrm{~s}$. The proposed method has a faster response and stronger robustness. With a designed bi-DSP suspension controller, this nonlinear control method was implemented on the Shanghai Urban Maglev Test Line (SUMTL) to validate its effectiveness and feasibility.
\end{abstract}

Key words: nonlinear control; electromagnetic suspension (EMS); maglev train; DSP

(C) 2011 JMT. All rights reserved.

\section{Introduction}

A $\mathrm{s}$ an electromagnetic suspension (EMS) system is nonlinear and open-loop unstable, it is usually linearized to build a linear model around the equilibrium point. Based on this linear model, a common linear control method is designed for suspension control, such as PID control. However, experiments show that the model will have poor performance when distant from the equilibrium point. To deal with this problem, active research has been carried out. Trumper et al. [1] compared the performance of linear and nonlinear digital control schemes in a well-controlled experimental environment. Liu et al. [2] and Joo et al. [3] designed nonlinear controllers, and made an experimental comparison between feedback linearizing control and classical state feedback control. Ref.[4] provides a gain scheduled control of magnetic suspension system. Shen [5] compared the performance of the $\mathrm{H} \infty$ controller, the sliding mode controller and the PID controller. All of the above controllers were implemented on a single EMS system for experimental purposes only. In this paper, a nonlinear control algorithm for EMS was proposed and implemented on the Shanghai Urban Maglev Test Line (SUMTL).

Received Jul. 1, 2011; revision accepted Aug. 26, 2011

*Corresponding author. E-mail: nmtcxjq@163.com (J.Q. XU) (C) 2011 JMT. All rights reserved doi: 10.3969/j.issn.2095-087X.2011.03.005

\section{Nonlinear feedback: linearizing math- ematic model}

An EMS system generally consists of several electromagnet modules, each of which has six degrees of freedom, i.e., lift, lateral motion, longitudinal motion, rolling, pitch, and yaw. By mechanically decoupling, EMS system control can be decomposed into a single electromagnet module control unit. Actually, the bogie and carriage of the maglev train are connected by an airspring (called the secondary spring). Due to the large difference between inherent frequency of the secondary spring and spectrum of the suspension close-loop control system, the effect of secondary spring on system dynamic performances can be ignored. The deformation response of the vehicle-guideway is treated as a disturbance force. A simplified single-electromagnet suspension system module is illustrated in Fig. 1.

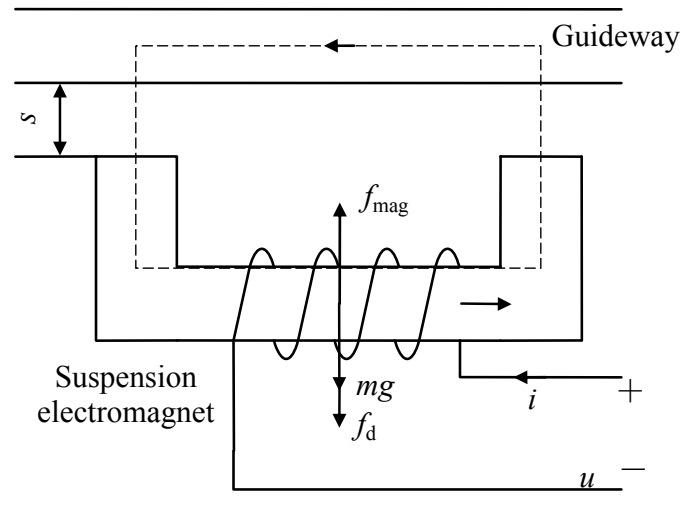

Fig. 1 Single-electromagnet suspension system module 
Here $m$ is the gross mass of bogie, carriage, load and electromagnet; $i$ is the electromagnet coil current; $s$ is the suspension airgap; $u$ is the electromagnet coil voltage; $f_{\text {mag }}$ is suspension force produced by electromagnet; and, $f_{\mathrm{d}}$ is the disturbance force.

One considers the relationship between the voltage and current of electromagnet and assumes the positive direction is vertically upward. The dynamic equation for the single-electromagnet suspension system is given as [2,6-7]

$$
\left.\begin{array}{l}
m \ddot{s}+f_{\text {mag }}=m g+f_{\mathrm{d}}, \\
u=R i+\frac{2 k i}{s}-\frac{2 k i \dot{s}}{s^{2}}, \\
f_{\text {mag }}=k\left(\frac{i}{s}\right)^{2},
\end{array}\right\}
$$

where the symbol $k=\mu_{0} N^{2} A / 4$ is the electromagnet constant; $N$ is the number of turns; $A$ is the valid pole area of coil; $R$ is the coil resistance; $\mu_{0}$ is the permeability of air.

\section{Nonlinear control algorithm design}

Fig. 1 shows that equation (1) expresses the motion characteristic of electromagnet. Normally, the value of the airgap $s$ can be directly derived from eddy-current sensor, and the value of coil current $i$ from Hall-type sensor. Thus, the three state variables, airgap $s$, current $i$ and airgap differential $\dot{s}$ are selected as control variables and set as

$$
x_{1}=s, x_{2}=\frac{\mathrm{d} x_{1}}{\mathrm{~d} t}, x_{3}=i
$$

Substituting them into (1) yields

$$
\left[\begin{array}{c}
\dot{x}_{1} \\
\dot{x}_{2} \\
\dot{x}_{3}
\end{array}\right]=\left[\begin{array}{c}
x_{2} \\
-\frac{k}{m}\left(\frac{x_{3}}{x_{1}}\right)^{2}+g \\
\frac{x_{2} x_{3}}{x_{1}}-\frac{R}{2 k} x_{3} x_{1}
\end{array}\right]+\left[\begin{array}{c}
0 \\
0 \\
\frac{x_{1}}{2 k}
\end{array}\right] u+\left[\begin{array}{c}
0 \\
\frac{f_{\mathrm{d}}}{m} \\
0
\end{array}\right] .
$$

When the high-speed current close-loop control is applied, the damping effect of coil inductance to current dynamic response can be ignored, and Eq.(2) can be simplified $[5,10]$ :

$$
\left[\begin{array}{l}
\dot{x}_{1} \\
\dot{x}_{2}
\end{array}\right]=\left[\begin{array}{c}
x_{2} \\
-\frac{k}{m}\left(\frac{i}{x_{1}}\right)^{2}+g+\frac{f_{\mathrm{d}}}{m}
\end{array}\right] .
$$

For system linearization, the following notations are introduced:

$$
z_{1}=x_{1}-s_{0}, z_{2}=x_{2}, w=g-\frac{k}{m} \frac{1}{x_{1}^{2}} i^{2}+\frac{f_{\mathrm{d}}}{m},
$$

where $s_{0}$ is a nominal value of air gap at the equilibrium point. By taking $w$ as an input of the linear control system, and substituting (4) into (3), we obtain the equivalent linear open-loop model of Eq.(3):

$$
\left[\begin{array}{l}
\dot{z}_{1} \\
\dot{z}_{2}
\end{array}\right]=\left[\begin{array}{ll}
0 & 1 \\
0 & 0
\end{array}\right]\left[\begin{array}{l}
z_{1} \\
z_{2}
\end{array}\right]+\left[\begin{array}{l}
0 \\
1
\end{array}\right] w \text {. }
$$

Eq.(5) shows that the open-loop system is controllable and can be stabilized by applying the state-feedback control method. Introducing a control rule $w=-\left(k_{1} z_{1}+k_{2} z_{2}\right)$ into (4), we obtain the corresponding current control law:

$$
i=x_{1} \sqrt{\frac{m k_{1} z_{1}+m k_{2} z_{2}+m g+f_{\mathrm{d}}}{k}},
$$

where the disturbance force $f_{\mathrm{d}}$ is calculated according to (1), and $f_{\text {mag }}$ is calculated from $f_{\text {mag }}=k(i / s)^{2}$ in (1) with the measured value of current $i$ and airgap $s$. $\ddot{s}$ can be obtained from accelerometer for it is equivalent to acceleration $a$; and gross mass $m$ is a known quantity.

Eq.(6) can be rewritten as

$$
\begin{gathered}
f_{\text {con }}=k\left(\frac{i}{s}\right)^{2}=k_{1}^{\prime}\left(s-s_{0}\right)+k_{2}^{\prime} \dot{s}+f_{\mathrm{d}}+f_{0}= \\
f_{\mathrm{p}}+f_{\mathrm{ds}}+f_{\mathrm{d}}+f_{0} .
\end{gathered}
$$

Correspondingly, the current control variable $i_{\text {con }}$ is given by

$$
i_{\text {con }}=s \sqrt{\frac{f_{\text {con }}}{k}},
$$

where

$$
k_{1}^{\prime}=\frac{m k_{1}}{k} ; k_{2}^{\prime}=\frac{m k_{2}}{k} ; f_{0}=m g
$$

Eq.(7) shows that the electromagnetic force $f_{\text {con }}$ should consist of four parts:

(1) To ensure the electromagnet works at a stable point (practical airgap $s$ at nominal value $s_{0}$ ), the control force for eliminating the static error of airgap is $f_{\mathrm{p}}=k_{1}^{\prime}\left(s-s_{0}\right)$.

(2) The damping force from differential calculation of airgap: $f_{\mathrm{ds}}=k_{2}^{\prime} \dot{s}$.

(3) To overcome the disturbance force, the antidisturbance magnetic force is equal to $f_{\mathrm{d}}$. 
(4) In addition to these three forces for dynamic adjustment, the static magnetic force is necessary to overcome the weight $\left(f_{0}=m g\right)$ of the EMS system.

From the analysis, unless the electromagnetic force balances the four forces illustrated above, the system will reach stability. The control diagram is shown in Fig. 2, where $i_{1}, a$, and $s$ are input variables which can be measured directly; $i_{1}$ is electromagnet coil current measured by sensor; $i_{\text {con }}$ is calculated control variable (the signal of desired current); $k_{1}^{\prime}$ and $k_{2}^{\prime}$ are variable parameters to adjust suspension stiffness and system damping.

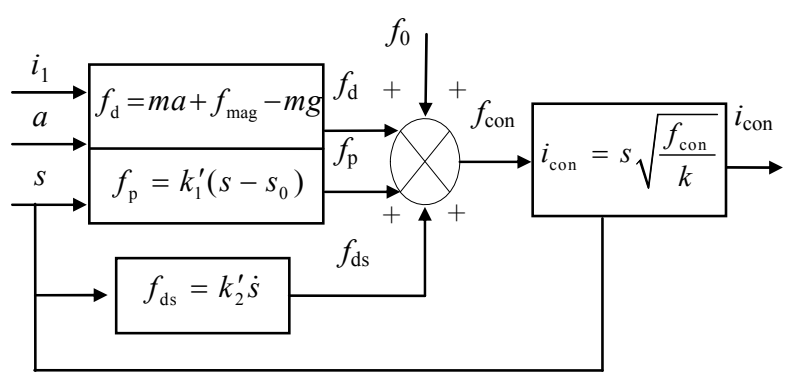

Fig. 2 Nonlinear control method diagram

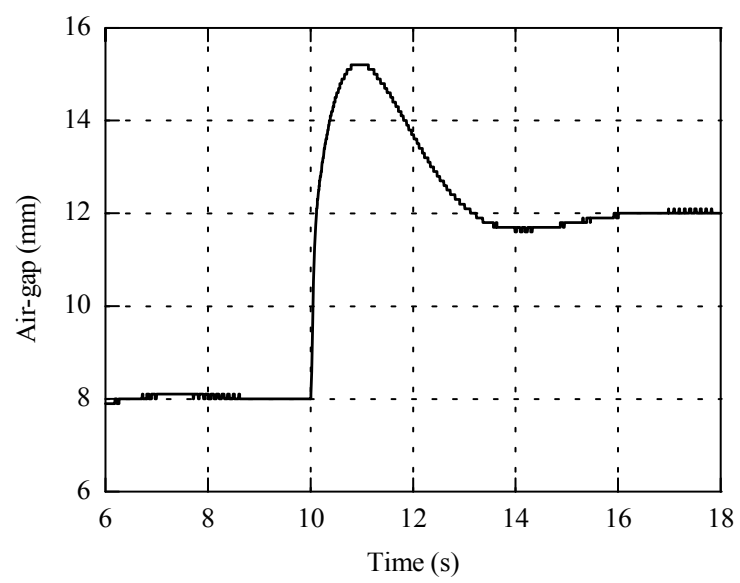

(a) System step response for a PID controller

\section{Simulation}

The following physical parameters are chosen in the simulation: $m=750 \mathrm{~kg}, N=340, R=0.4, s_{0}=0.008 \mathrm{~m}$, $A=0.028 \times 0.75 \mathrm{~m}^{2}$. Fig. 3 (a) shows the system response to an airgap step disturbance of $4 \mathrm{~mm}$ (nominal airgap $s_{0}$ is changed from 8 to $12 \mathrm{~mm}$ abruptly) when the system is in steady state, which is based on the classical PID controller. The PID controller is designed as follows [8]:

$$
G(s)=k_{\mathrm{p}}+k_{i} / s+k_{\mathrm{d}} s .
$$

Referring to [8], we choose the optimized parameters as follows:

$$
k_{\mathrm{p}}=550, k_{i}=250, k_{\mathrm{d}}=190 .
$$

Fig. 3 (b) shows the response of the proposed nonlinear controller to the same step disturbance. The simulation results show that, for a PID controller, the overshoot of the system response to an airgap step disturbance is about $3 \mathrm{~mm}$, and the transient time is $6 \mathrm{~s}$; however, for the proposed nonlinear controller, there is no overshoot and transient time within $2 \mathrm{~s}$. This verifies that the proposed controller has a faster response and stronger robustness than the PID controller.

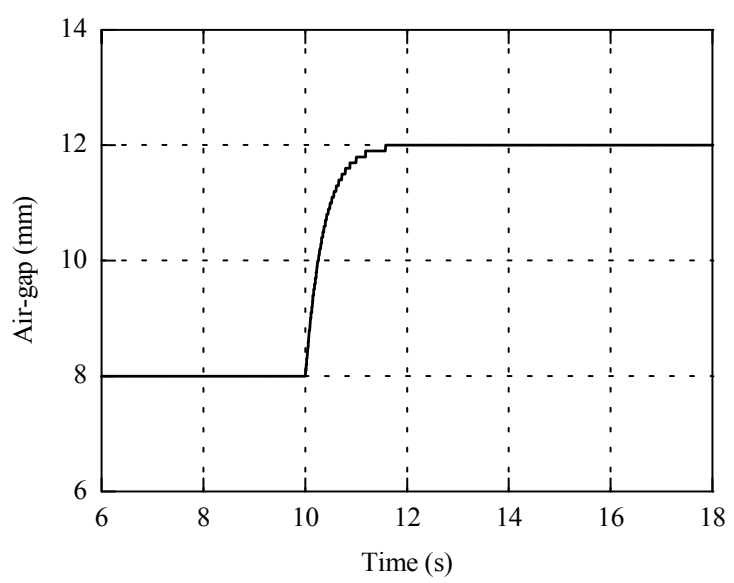

(b) System step response for the proposed nonlinear controller

Fig. 3 System responses with different controllers

\section{Experimental configuration}

\subsection{Control circuit structure}

The control circuit for the proposed control algorithm is constructed based on a bi-DSP structure shown in Fig. 4, where the model of DSP1 and DSP2 is TMS320F2812. Double Port Random Memory chip achieves the parallel data communication between the two DSPs. The address logic among DSP1, DSP2, and peripheral chips is controlled by CPLD. The high- precision multi-parallel sampling A/D chip is extended. And CAN interface for communication between the suspension controller and upper computer is deployed. DSP1 performs sampling and filtering of the airgap, acceleration, and current signal, and calculates the differential value of the airgap signal. Before being sampled by $\mathrm{A} / \mathrm{D}$, the three signals need to be filtered through Analog Anti-aliasing Filter (AAF); DSP2 performs the proposed control algorithm, calculates the desirable current $i_{\text {con }}$, and then generates the PWM pulse control signal. In addition, DSP2 can communicate with the upper computer through the CAN interface to implement the 
setup of control parameters and the upload of state information. The advantages of bi-DSP hardware structure lie in the rapidity in sampling and follow-up processing, the decrease in signal delay, and the high efficiency in complicated calculation, such as nonlinear control algorithm, differential, square/extraction, and scaling, etc.

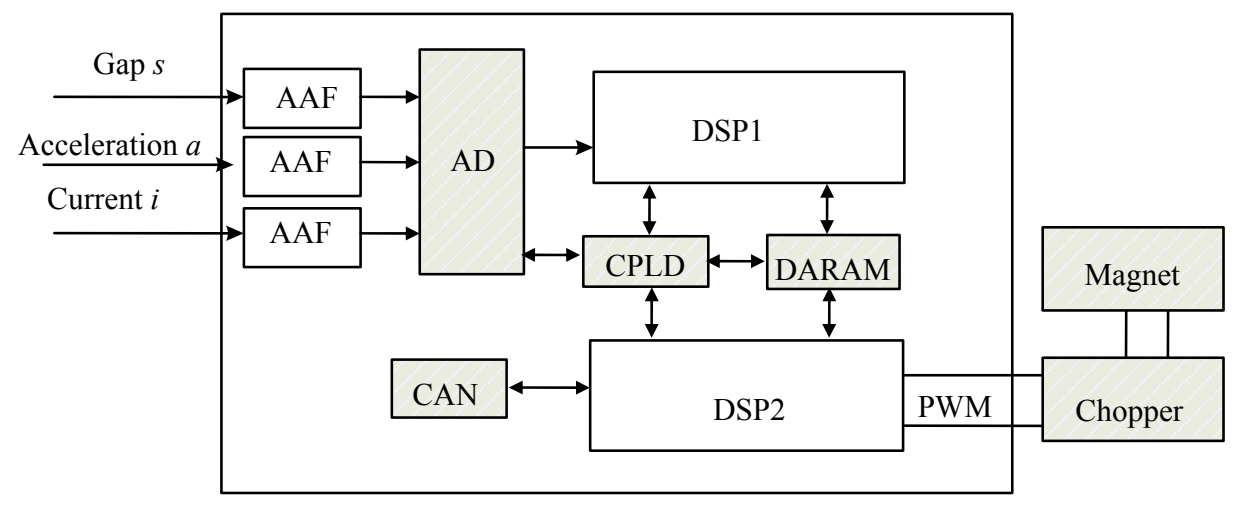

Fig. 4 Block diagram for bi-DSP suspension control

\subsection{Control software}

The control software includes two parts: signal processing software in DSP1 and control algorithm software in DSP2. With debugging experiences, we setup the interruption cycles of DSP1 and DSP2, respectively, $100 \mu \mathrm{s}$ and $500 \mu \mathrm{s}$.

\section{Experimental results}

With the designed suspension controller, the suspension and propulsion experiment was carried out on the Maglev train at SUMTL. This Maglev train consists of three sections and each section is supported by five suspension bogies. At each side of a suspension bogie, one suspension electromagnet is assembled separately, and each electromagnet has two control circuits, which are controlled by two of the designed controllers. In total, there are twenty suspension controllers in one section. In the experiment, the 3-section train can stably pass those critical track segments with a $50 \mathrm{~m}$ horizontal curve, $70 \mathrm{~m}$ horizontal curve, $1500 \mathrm{~m}$ vertical curve, and the steel guideway switch. The maximal speed of the train is $100 \mathrm{~km} / \mathrm{h}$ and the suspension fluctuation is within $\pm 2 \mathrm{~mm}$.

Figs. 5-7 show the experimental results. Fig. 5 shows the airgap data of a suspension point at the speed of $90 \mathrm{~km} / \mathrm{h}$, where the peaks reflect the timing when the airgap sensor detects the guideway joint gaps. Fig. 6 shows the airgap data of a suspension point when the train was passing along the track of $70 \mathrm{~m}$ horizontal curve at $35 \mathrm{~km} / \mathrm{h}$ with the proposed nonlinear controller. In contrast, Fig. 7 shows the airgap data of suspension points under the same working conditions but with a traditional PID controller.

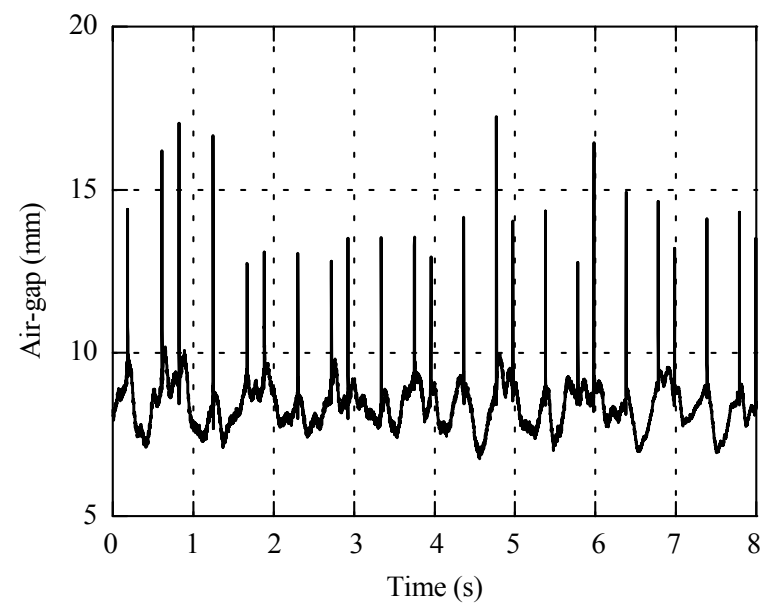

Fig. 5 Suspension airgap signal at $90 \mathrm{~km} / \mathrm{h}$ using the proposed nonlinear controller

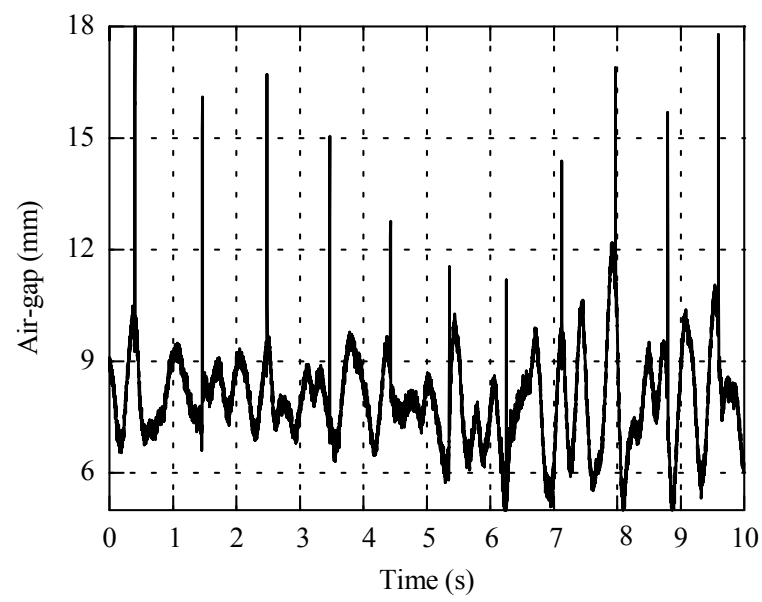

Fig. 6 Suspension airgap signal passing by a $70 \mathrm{~m}$ curve using the proposed nonlinear controller 


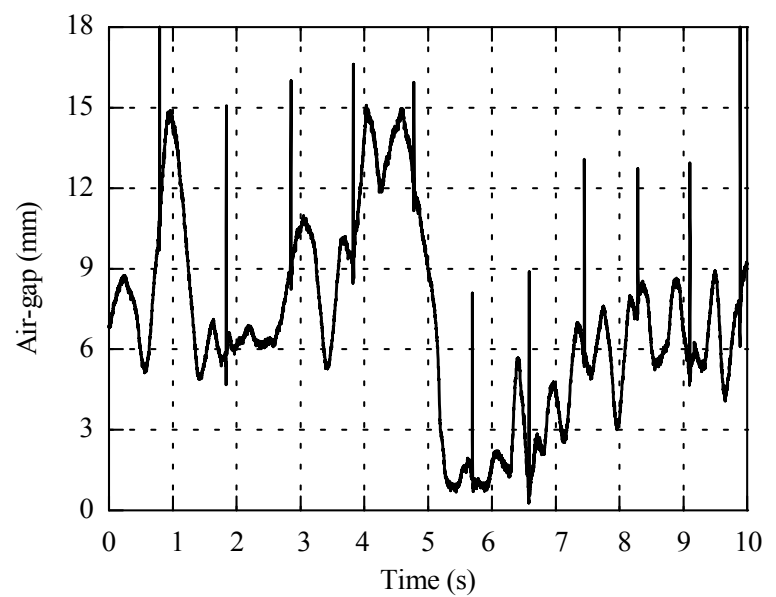

Fig. 7 Suspension airgap signal passing by a $70 \mathrm{~m}$ curve under PID control

When the train is passing the curve, the excitation model is changed, apparently due to the relative position deviation between the electromagnet and F-shaped rail, and further the relative position change between the carriage and bogie leads to a dynamic change of load at suspension point. The controller has to regulate the electromagnet current quickly and precisely so that the system parameters change will not affect suspension stability. From Fig. 7, it is found that the traditional PID controller cannot real-time adjust the control variables when system parameters change, which results in large airgap fluctuation of about $\pm 6 \mathrm{~mm}$ and the system stability is damaged: the electromagnets and skids touch the " $F$ " shaped track. However, with the proposed nonlinear control, the real-time adjustment of control variables can be achieved, avoiding large airgap fluctuation (airgap fluctuation limited within $\pm 3 \mathrm{~mm}$ as shown in Fig. 6). The experimental results prove that the proposed nonlinear control can greatly improve the system robustness and adaptability to guide parameter change.

\section{Conclusion}

Based on the dynamic model of a single electromagnet EMS system, a nonlinear control algorithm is proposed. Furthermore, a bi-DSP suspension controller is designed and implemented according to this control algorithm, and the controller is applied on the Maglev train on SUMTL. Field tests on the test line validate the adaptability of this control method to all working conditions. Compared with the traditional PID control, this proposed control method is more effective and feasible, which is significant in the optimization of suspension control system. In the future, we will address aspects of the controller such as parameters optimization, decoupling control between two control circuits on one magnet, and reliability analysis.

\section{References}

[1] D.L. Trumper, S.M. Olson, P.K. Subrahmanyan, Linearizing control of magnetic suspension systems, IEEE Transactions on Control Systems Technology, 1997, 5(4): 427-438.

[2] H.K. Liu, X.H. Shi, W.S. Chang, Nonlinear control of maglev, Systems Control Engineering of China, 2007, 14(5):455-457(in Chinese).

[3] S.J. Joo, J.H. Seo, Design and analysis of the nonlinear feedback linearizing control for an electromagnetic suspension system, IEEE Transactions on Control Systems Technology, 1997, 17(5): 135-144.

[4] L.Y. Kim, K.H. Kim, Gain scheduled control of magnetic suspension systems, In: American Control of Conference, Baltimore, 1994: 3127-3131.

[5] J.C. Shen, $\mathrm{H} \infty$ control and sliding mode control of magnetic levitation system, Asian Journal of Control, 2002, 4(3): 333-340.

[6] H. Jiang, J.S Lian, The dynamic model and control of single-magnet suspension systems, Journal of Southwest Jiaotong University, 1992, 83(1): 59-67 (in Chinese).

[7] J. Bao, K.L. Zhang, Research of electromagnetic suspension system of single magnetic, Computer Automated Measurement \& Control, 2003, 11(11): 863-865 (in Chinese).

[8] L.H. Liu, Design of PID controller for EMS-type magnetic levitation vehicle, Journal of Zhejiang Wanli University, 2005, 18(2): 99-102 (in Chinese).

[9] Z.Q. Long, H.J. Hong, X.B. Zhou, Research of nonlinear control for maglev train, Control Theory \& Applications, 2003, 20(3): 399-402 (in Chinese).

[10] H.B. Shang, Digital Control Method of Electromagnetic Suspension System and Its Realization [Dissertation], Chengdu: Southwest Jiaotong University, 2003 (in Chinese).

[11] Y.Q. Deng, S.H. Luo, Stability research and simulation of a single magnetic system, Electric Locomotives \& Mass Transit Vehicles, 2005, 28(5): 44-46 (in Chinese).

[12] C.T. Hu, S.L. Chen, Exact linearization of magnetic bearing system, IEEE Transactions on Control Systems Technology, 2002, 10(4): 619-625.

[13] L.R. Hunt, R. Su, G. Meyer, Global transformation of nonlinear systems, IEEE Trans Automatic Control, 1983, 28(1): 24-30.

[14] E. Gottzein, B. Lange, Magnetic suspension control system for the MBB high speed train, Automatic, 1975, 11(6): 271-284.

[15] T. Namerikawa, M. Fujita, F. Matsummra, Wide area stabilization of a magnetic bearing using exact linearization, Electrical Engineering, 1999, 128(2): 53-62.

[16] H.K. Liu, A.M. Hao, W.S. Chang, Nonlinear PID control of magnetic suspension systems, Control Engineering of China, 2007, 14(6): 653-656.

[17] Y.G. Li, W.S. Chang, Z.Q. Long, Guideway resonance vibration and levitation control system design of an EMS maglev vehicle, Transaction of National University of Defense Technology, 1999, 21(2): 93-96.

(Editor: Yao ZHOU) 